\title{
Inertial Response from Offshore Wind Farms Connected through DC Grids
}

\author{
Inmaculada Martínez Sanz, Student Member, IEEE, Balarko Chaudhuri, Senior Member, IEEE, \\ and Goran Strbac, Member, IEEE
}

\begin{abstract}
Inertial response from remote offshore wind farms (WFs) connected through point-to-point DC links relies on emulation of the onshore $\mathrm{AC}$ system frequency variation at each offshore wind turbine generator (WTG). This is not straightforward for a DC grid interconnecting WFs to multiple onshore AC systems. To address this problem this paper builds up on a communication-less approach previously reported for provision of frequency services from offshore WFs connected through a DC grid. A weighted frequency scheme is adopted which relies on the fiber optic link embedded within the sub-sea cables for fast communication of onshore frequency variations up to the offshore converters. The effectiveness of the proposed approach is shown analytically and also through a case study on a 4 terminal DC grid interconnecting an offshore wind farm and two onshore AC systems.
\end{abstract}

Index Terms-Inertial response, offshore wind farm, DC grid, droop control, frequency support, voltage source converter (VSC).

\section{INTRODUCTION}

W IND power is likely to replace a significant proportion of fossil-fuel based generation in countries like the UK where around $17 \mathrm{GWs}$ of offshore wind power capacity are planned to be installed by 2020 [1]. Unlike conventional synchronous power plants, wind farms (WFs) do not naturally contribute to system inertia. Replacement of a significant amount of synchronous machines with wind generation would therefore cause a drastic reduction in the effective inertia of future power systems. This would result in large frequency excursions and high rates of change of frequency (RoCoF) after an event like the loss of a generating unit, impacting the system security and stability [2]. In response to frequency variations, the WFs could be made to provide inertial response through proper control. For example, following a dip in AC system frequency the wind turbines could be slowed down to release the kinetic energy stored in the turbine blades to help restore the system frequency [3], [4]. Inertial support from large wind farms (WFs) is going to be critical for secure operation of low inertia systems of the future [2].

For onshore WFs and AC connected offshore WFs, individual wind turbine generators (WTGs) are synchronously connected to the main grid and can directly sense the variations in AC system frequency to provide inertial support. However, remote offshore WFs are connected through a DC link, which

I. Martínez Sanz, B. Chaudhuri and G. Strbac are with the Department of Electrical and Electronic Engineering, Imperial College London, London, SW7 2AZ U.K. (e-mail: im1309@imperial.ac.uk; b.chaudhuri@imperial.ac.uk; g.strbac@imperial.ac.uk). The authors acknowledge the financial support and technical inputs from National Grid U.K. decouples the WF from the onshore AC system. Frequency variations in onshore systems can no longer be sensed directly by each WTGs. In such cases, the measured onshore frequency variations can be communicated up to the offshore converter station to set the frequency of the WF collection network accordingly [5]. Alternatively, in a communication-less scheme, the onshore frequency variation could be emulated at the WF through a proportional variation in the DC link voltage using a voltage-frequency droop at both on- and offshore converters [6]-[8]. For a point-to-point DC connection between the WF and the onshore grid, either of the above approaches ensures that individual WTGs are able to sense the emulated variation in the onshore grid frequency and provide inertial support.

In the future, WFs are likely to be connected to more than one onshore AC systems through a DC grid based on voltage source converter (VSC) technology [9] to increase the flexibility in operation and security of supply. For example, a North-Sea grid [10] and an European Supergrid [11] are envisaged to allow effective sharing of intermittent renewable energy sources between the European countries. Emulation of onshore grid frequencies at each WTG (required to provide inertial support) is not straightforward for offshore WFs connected to multiple onshore AC systems through a DC grid. The challenge is to deal with multiple frequency variations in different AC systems which could have opposite trends [12]. Onshore converters within a DC grid can be controlled to allow exchange of frequency support among the host onshore AC systems. Use of frequency-voltage $(f-V)$ droop control together with existing power-voltage $(P-V)$ droop control for autonomous power sharing [13] has been proposed in [14], [15]. An alternative scheme using direct communication between the AC areas is reported in [16].

However, there is hardly any paper on the frequency support provision from offshore WFs connected through DC grids. Reference [17] is the first and arguably the only paper that deals with this specific issue: it proposes a communication-less scheme based on use of droop control at both on- and offshore converters. The onshore converter switches from $P-V$ droop to $f-V$ droop control when a frequency change is detected at the corresponding $\mathrm{AC}$ system. Thus frequency support from offshore WFs could be provided across a DC grid together with exchange of frequency services among onshore AC systems. However, there could be potential issues with power reference tracking and proper emulation of onshore frequency deviations at the offshore WFs.

Building up on similar ideas as in [17] we propose a control strategy that uses the existing high-speed fiber optic 
link embedded within a sub-sea cable for communicating the onshore frequency variations up to the offshore converter stations of a DC grid. The frequency references of the offshore converters are set to follow a weighted sum of the negative part of the onshore system frequency variations so as to respond only to under frequency events. Thus the onshore frequency variations are emulated at the individual WTGs through the WF collection grid triggering their inertial response. The strategy works in conjunction with the existing $P-V$ and $f-V$ droop controllers employed at the onshore converter stations for autonomous power sharing and exchange of frequency support. The effectiveness of the proposed strategy is evaluated both analytically and through a case study on a 4-terminal DC grid interconnecting two host AC systems and an offshore WF.

\section{DC GRID CONVERTER CONTROL}

\section{A. Control of Onshore Converters}

As a natural extension to point-to-point HVDC links, all but one converters in a DC grid can be controlled to maintain their respective reference power while the remaining converter controls the DC link voltage, acting as a slack converter. In case of a converter outage, only the slack converter will take up the entire share of the resulting power imbalance. Moreover, the outage of the slack converter will lead to the shut down of the DC grid. For this reason use of power-voltage $(P-V)$ droop in each converter is recommended as it allows all the converters to operate in DC link voltage control mode in order to achieve autonomous power sharing [18]. The value of the droop constant influences the sharing of the power imbalance between the converters [13].

Including an additional frequency-voltage $(f-V)$ droop control on top of the $P-V$ droop allows exchange of frequency reserves between the $\mathrm{AC}$ systems connected to the DC grid [14], [15]. Figure 1 shows the single line diagram of the onshore converter with decoupled control of the active and reactive power. Both $P-V$ and $f-V$ droop controllers are considered in the active power control loop, which can be expressed mathematically as:

$$
V_{d c}=V_{d c}^{r e f}-k_{p}\left(P_{d c}-P_{d c}^{r e f}\right)+k_{f}\left(f-f^{r e f}\right)
$$

where $k_{p}$ and $k_{f}$ are the $P-V$ and $f-V$ droop constants, respectively. Note that the measured power is considered positive if the station acts as a rectifier.

The presence of a $f-V$ droop not only allows exchange of frequency support between the onshore AC systems but is also necessary to efficiently transfer the inertial response from the offshore WFs connected through a DC grid. The additional power injected by the offshore WFs would be countervailed by the onshore converters if only $P-V$ droop control is in place, i.e. the power injected by the onshore converters would change to try to balance the surplus power within the DC grid, reducing the inertial support from the WFs.

\section{B. Control of Offshore Converters}

The offshore converters connecting the offshore wind farms to the DC grid are required to be controlled to transfer all the power the WFs are producing on to the DC grid. Typically,

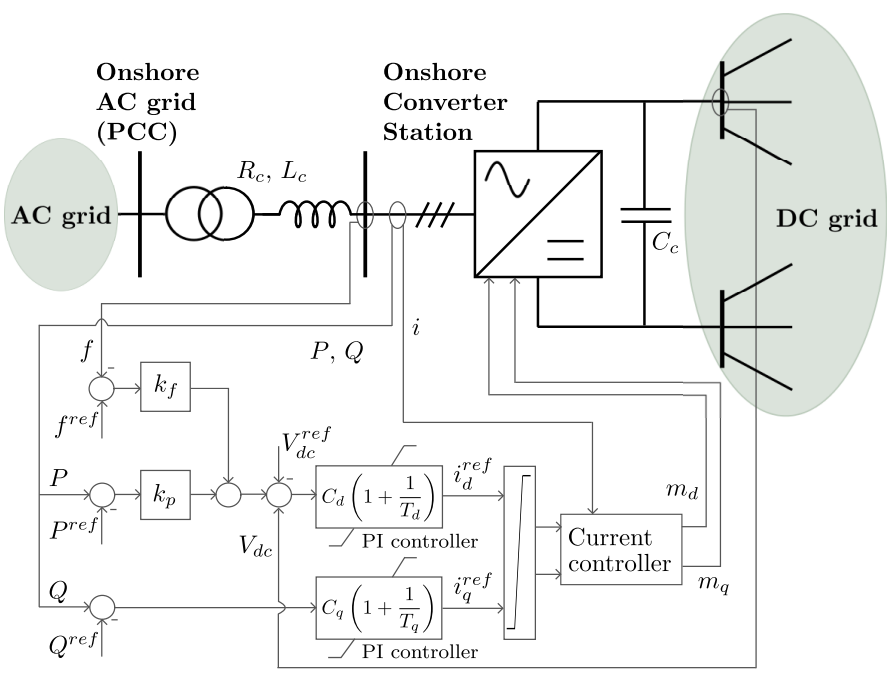

Fig. 1. Control of onshore converter.

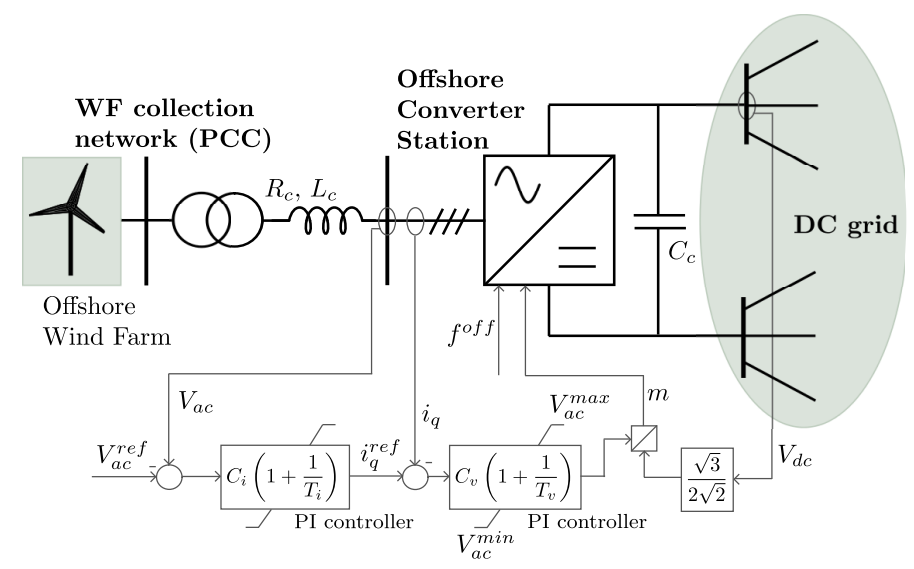

Fig. 2. Control of offshore converter.

the offshore converters act as stiff AC voltage sources which tightly regulate the offshore $\mathrm{AC}$ voltage $\left(V_{a c}\right)$ at a given frequency $\left(f^{\circ f f}\right)$ as shown in Fig. 2 [19]. The offshore frequency can be altered without significantly affecting the operation of the WF: as it is unlikely that loads will be connected directly to the offshore grid, variable frequency operation of the system is possible [20].

\section{Wind Turbine Generator (WTG) CONTROL}

\section{A. Basic Control}

Variable speed wind turbines with fully rated converter (FRC) and permanent magnet synchronous generator are considered in this study [21]. The control strategy for the FRC WTGs is shown in Fig. 3. This includes the control loops for each converter, maximum power point tracking (MPPT) and pitch angle control to regulate the power extracted from the wind $\left(P_{W}\right)$ which can be expressed as:

$$
P_{W}=\frac{1}{2} \pi \rho R^{2} v_{w}^{3} C_{p}(\beta, \lambda)
$$

where $\rho$ is the air density, $R$ is the radius of the turbine, $v_{w}$ is the wind speed and $C_{p}$ is the power coefficient. $C_{p}$ 


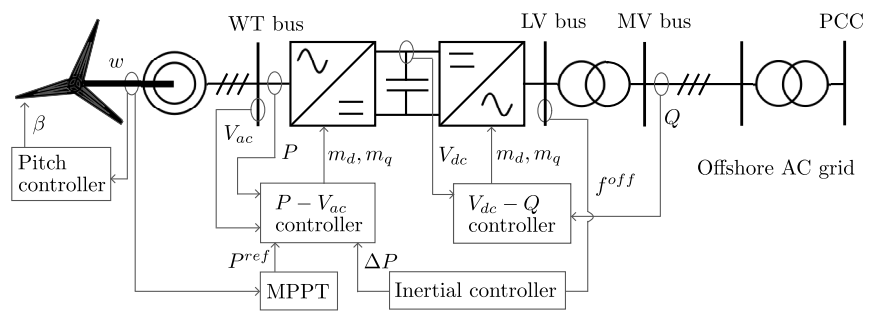

Fig. 3. Wind turbine generator (WTG) control.

is dependent on the pitch angle $\beta$ and the tip speed ratio $\lambda$, defined as $\lambda=\omega_{r} R / v_{w}$, where $\omega_{r}$ is the rotational speed of the turbine blades.

For low wind speed conditions, the wind turbine operates in the so called sub-rated regime. The MPPT control adjusts the rotor speed to ensure that the turbine operates at the optimal tip speed ratio $\lambda_{\text {opt }}$, extracting the maximum power from the wind. The pitch angle is kept to $0^{\circ}$, which corresponds to the maximum power coefficients. Alternatively, for wind speeds above the rated one, the turbine operates in the rated regime with an increased pitch angle $(\beta)$ to limit the turbine speed and output power at their rated values.

\section{B. Inertial Control}

The WFs can provide frequency support in two ways. A possibility is to operate according to a de-loaded optimum power extracting curve [22]. Alternatively, temporary overproduction can be achieved through the release of the kinetic energy stored in the turbine blades by slowing the speed of the turbine [23] or pitching the blades [24]. In this paper we consider that the inertial response of the wind farm does not depend on de-loaded operation.

There are several mechanism for extracting inertial response from WTGs [3], [25], [26]. Here we employ the derivative of the frequency variations to modify the power generated from the WTGs using a first order filter after the $d \Delta f / d t$ input [3].

$$
\Delta P=-K_{I} \frac{d \Delta f}{d t}
$$

This particular work focuses specifically on inertial response from offshore WFs connected through DC grids. However, the proposed framework is general and also applies to primary frequency support from WFs with power reserve margin [27].

\section{EMUlation OF ONSHORE FREQUENCY VARIATIONS AT OFFShORE Wind Turbine GENERATORS (WTGS)}

In this section two approaches for emulating the onshore frequency variations at the individual offshore WTGs across a DC grid are presented.

\section{A. Communication-Less Scheme (CLS)}

In order to allow WFs connected through a point-to-point DC link to participate in frequency control, communicationless schemes have been proposed [6]-[8]. The onshore converter produces a change in DC link voltage proportional to the onshore frequency variation using a frequency-voltage ( $f$ $V$ ) droop. Using a similar $V-f$ droop, the offshore converter sets the frequency of the corresponding offshore WF collection network in proportion to the sensed DC voltage variation at the offshore end:

$$
f^{o f f}=f^{r e f}+k_{o f f}\left(V_{d c}-V_{d c}^{r e f}\right)
$$

where $k_{o f f}$ is a proportional term. This results in perfect emulation of onshore frequency at each WTG, if the droop constants are chosen appropriately. A similar scheme was proposed in [17] in the context of offshore WFs connected to multiple onshore AC systems through a DC grid, henceforth referred to as Communication-Less Scheme (CLS). The CLS changes the frequency of the offshore WF according to (4). However, as a DC grid can have different topologies and several droop controllers, it is difficult to properly emulate the onshore frequency variations at each WF collection network. This can be explained from the expression for DC voltage at the offshore converter as derived next.

Let us consider a DC grid with $n$ converter stations where the $n^{\text {th }}$ one is the offshore converter station connected to an offshore WF while the remaining $(n-1)$ are onshore converter stations. Only one offshore converter is considered here for simplicity without any loss of generality.

The current injected in the DC network by any converter $i$ can be expressed as:

$$
I_{d c_{i}}=\sum_{j=1, j \neq i}^{n} G_{i j}\left(V_{d c_{i}}-V_{d c_{j}}\right)
$$

where $G_{i j}$ is the conductance of the line connecting stations $i$ and $j$. We can re-write (5) for the offshore converter $n$ as:

$$
I_{d c_{n}}=G_{n n} V_{d c_{n}}-\sum_{j=1}^{n-1} G_{n j} V_{d c_{j}}
$$

where $G_{n n}=\sum_{j=1}^{n-1} G_{n j}$. For a fixed wind speed, the offshore converter injects a constant power $P_{d c_{n}}^{o f f}$ into the DC grid through a current $I_{d c_{n}}$ given by:

$$
I_{d c_{n}}=P_{d c_{n}}^{o f f} / 2 V_{d c_{n}}
$$

From (7), changes in $I_{d c_{n}}$ and $V_{d c_{n}}$ from their nominal values $I_{d c_{n}}^{0}$ and $V_{d c_{n}}^{0}$ can be approximated using the Taylor series as:

$$
I_{d c_{n}} \approx I_{d c_{n}}^{0}-\frac{I_{d c_{n}}^{0}}{V_{d c_{n}}^{0}}\left(V_{d c_{n}}-V_{d c_{n}}^{0}\right)
$$

By substituting (8) in (6), we derive an expression for the DC link voltage at the offshore converter station:

$$
V_{d c_{n}}=\frac{1}{\left(G_{n n}+I_{d c_{n}}^{0} / V_{d c_{n}}^{0}\right)}\left(2 I_{d c_{n}}^{0}+\sum_{j=1}^{n-1} G_{n j} V_{d c_{j}}\right)
$$

Grouping the constant terms and including the expression in (1) for $V_{d c_{j}}$, the offshore DC voltage can be expressed in terms of the variables in the $n-1$ onshore converters:

$\left.V_{d c_{n}}=a_{n}+\sum_{j=1}^{n-1} b_{n_{j}}\left[V_{d c_{j}}^{r e f}-k_{p_{j}}\left(P_{d c_{j}}-P_{d c_{j}}^{r e f}\right)+k_{f_{j}}\left(f_{j}-f_{j}^{r e f}\right)\right)\right]$ 
where $a_{n}$ and $b_{n_{j}}$ are constants.

From (10) and (4) it can be seen that several factors, especially the term $k_{p_{j}}\left(P_{d c_{j}}-P_{d c_{j}}^{r e f}\right)$, disturb the proportionality between onshore and offshore frequency variations. To deal with this, the CLS distinguishes between normal operation mode (only $P-V$ droop control in place, $k_{f_{j}}=0$ ) and disturbed operation mode (only $f-V$ droop control in place, $k_{p_{j}}=0$ ) [17]. The disturbed mode is activated in a converter if the corresponding onshore frequency deviation is outside a certain dead-band (e.g. $\pm 20 \mathrm{mHz}$ ). This allows the provision of frequency services across the DC grid. However, there could be potential issues with power reference tracking and proper emulation of onshore frequency deviations at the offshore WFs due to the following reasons, also illustrated through simulation results later in Section VII-A:

- In normal operation, a change in power reference $\left(P_{d c}^{r e f}\right)$ command to vary the injections to/from the DC grid will disturb the frequency of the interconnected AC systems, triggering the disturbed operation mode [17]. In this condition the power through the converter is not allowed to follow the reference command, as the $P-V$ droop control is taken out of operation and the $f-V$ droop control aims to maintain the frequency of the system.

- The offshore DC voltage in (10) does not correspond to a proper weighted sum of onshore frequency variations, and the relative importance of each AC system frequency variation would be highly influenced by the DC grid topology and line resistances. In addition, a frequency event would not be reflected properly if each $\mathrm{AC}$ area is in a different operating mode (normal or disturbed [17]) or in the case of DC side disturbances (e.g. converter outage) that may lead to different frequency trends in the interconnected AC systems, as pointed out in [12].

\section{B. Weighted Frequency Scheme (WFS)}

Building up on the CLS, an alternative strategy that relies on fast communication of the onshore frequency variations up to the offshore converters is proposed in this paper. The existing high speed fibre optic link embedded within a sub-sea DC cable is used for communication without any extra investment. The offshore frequency is set to follow the weighted sum of the onshore $\mathrm{AC}$ system frequencies which is eventually reflected in the individual WTGs through the WF collection network. Thus the onshore frequency variations are properly emulated at each WTG, triggering their inertial response. Note that presence of fibre optics communication in every cable of the DC grid is not required, but there should exist at least one communication path from each onshore AC system to each offshore WF to ensure proper emulation of the frequency event in the offshore grid. The frequency in the offshore grid is shown in Fig. 4 and can be expressed as:

$$
f^{o f f}=f^{r e f}+\sum_{j=1}^{n_{\text {on }}} g_{j}\left(f_{j}-f_{j}^{r e f}\right)
$$

where $g_{j}$ is a weight term for each $j=1,2, . ., n_{\text {on }}$ corresponding to the onshore converter stations. We consider that the usual practice for the wind turbines (WTs) is to provide inertial

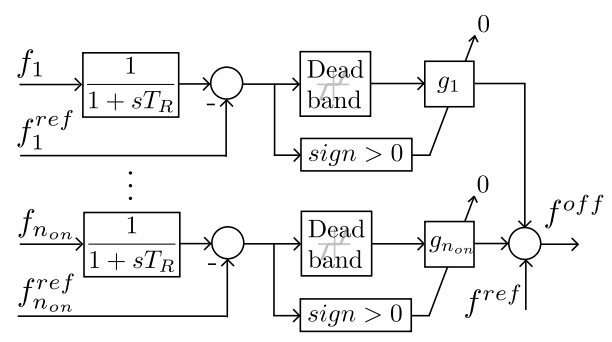

Fig. 4. Weighted Frequency Scheme (WFS) to emulate onshore frequency variations at offshore wind farms (WFs).

support by reducing the turbine speed, which releases the stored kinetic energy to compensate for the lack of available power and thus support a low frequency event in the AC grid. Accordingly, as WFs are meant to provide inertial response for the under-frequency events only, the weight $g_{j}$ is set to zero if the frequency variation in $j^{\text {th }} \mathrm{AC}$ system $\left(f_{j}-f_{j}^{r e f}\right)$ is positive. Additionally, the weight coefficients are chosen such that $\sum g_{j}=1$. Situations in which the wind farm has to reduce its power output (i.e. WT speeding up and absorbing energy) that occur during fault-ride through (FRT) and periodic modulation of the WT power output for power oscillation damping are out of the scope of this study. A worst-case time delay $T_{R}=0.1 \mathrm{~s}$ is considered to model the latency in communication of onshore frequencies variations. In reality, this fiber optic communication delay would be much less (of the order of 1-10 ms) for most of the time [28]. A dead band $( \pm 10 \mathrm{mHz})$ is included to avoid responding to ambient frequency deviations.

It was shown in [12] that there are two distinct patterns of dynamic variations of the onshore frequencies in response to AC-side and DC-side disturbances. The onshore frequencies vary similarly for AC-side disturbance while, for a DC-side disturbance, the frequency of the affected system (where the event took place) varies in the opposite direction compared to the rest of the AC systems. Possible masking effect in the weighted sum due to opposite trends in onshore frequencies is accounted for by setting the corresponding weights to zero to respond only to under-frequency events (as described above). Clipping the positive variations in frequency $\left(f_{j}-f_{j}^{r e f}\right)$ in the CLS approach would affect the exchange of frequency support between onshore AC systems.

As a final remark, a combination of both CLS and WFS strategies could be exercised for certain scenarios, e.g. considering CLS to emulate frequency variations of onshore systems connected point-to-point in the WF offshore grid.

\section{INERTIAL SUPPORT USING WEIGHTEd FREQUENCY SCHEME (WFS)}

An analytical formulation is presented in this section to show the effectiveness of WFS in reducing the negative frequency deviation in the onshore AC systems.

We consider a generic framework in which each AC system $i$ is connected to the DC grid by $m_{i}$ converter stations and where the total number of AC systems is $N$. Equation (1) for 
the onshore converter $j$ expressed in small-signal form is:

$$
\Delta P_{d c_{j}}=\Delta P_{d c_{j}}^{r e f}-\frac{1}{k_{p_{j}}} \Delta V_{d c_{j}}+\frac{k_{f_{j}}}{k_{p_{j}}} \Delta f_{j}
$$

For the offshore converter $j$ we derive a similar expression by using (3) and the offshore frequency defined by WFS in (11):

$$
\Delta P_{d c_{j}} \approx-K_{I} \frac{d \Delta f_{j}^{o f f}}{d t}=-K_{I} \sum_{j=1}^{n_{o n}} g_{j} \frac{d \Delta f_{j}}{d t}
$$

The total number of converters in the system is $n$, of which $n_{o n}$ are located onshore and $n_{o f f}$ offshore connecting the WFs. Note that $\sum_{i=1}^{N} m_{i}=n_{o n}$ and $N \leq n_{o n}$. If we neglect the change in DC grid losses, the sum of the exchanged power between the $n$ converters of the DC grid is zero:

$$
\sum_{j=1}^{n} \Delta P_{d c_{j}}=\sum_{j=1}^{n_{\text {off }}} \Delta P_{d c_{j}}+\sum_{j=1}^{n_{\text {on }}} \Delta P_{d c_{j}}=0
$$

Considering that voltage drops at all converter terminals are comparable, we can derive an expression for DC voltage variations using (12) and (14):

$$
\Delta V_{d c}=\sum_{j=1}^{n_{o n}} k_{p_{j}}\left(\sum_{j=1}^{n_{o f f}} \Delta P_{d c_{j}}+\sum_{j=1}^{n_{o n}} \Delta P_{d c_{j}}^{r e f}+\sum_{j=1}^{n} \frac{k_{f_{j}}}{k_{p_{j}}} \Delta f_{j}\right)
$$

Employing a classical first order power system model for frequency regulation studies, the power balance for each $\mathrm{AC}$ grid $i$ is given by:

$$
2 H_{i} \frac{d \Delta f_{i}}{d t}+D_{i} \Delta f_{i}=\Delta P_{G_{i}}-\Delta P_{L_{i}}-\sum_{i=1}^{m_{i}} \Delta P_{d c_{i}}
$$

where $P_{L_{i}}$ is the total active power demand in system $i$ and $P_{G_{i}}$ is the total generated power. If $R_{i}$ denotes the aggregated response of the governors in system $i$, it yields $\Delta P_{G_{i}}=-R_{i} \Delta f_{i}$.

Substituting (12), (13) and (15) in (16) and considering, for simplicity, the same constant $K_{I}$ in the control of all the offshore WFs, we obtain the following expression:

$$
\begin{gathered}
\overbrace{\left(2 H_{i}+\sum_{i=1}^{m_{i}} q_{i} n_{o f f} K_{I} g_{i}\right)}^{H_{i}^{\prime}} \frac{d \Delta f_{i}}{d t}+\Delta P_{L_{i}}= \\
-\overbrace{\left(R_{i}+\sum_{i=1}^{m_{i}} \frac{k_{f_{i}}}{k_{p_{i}}}\left(1-q_{i}\right)+D_{i}\right)}^{C_{i}} \Delta f_{i} \\
+\sum_{i=1}^{m_{i}} q_{i} \overbrace{\sum_{l=1, l \neq i}^{n_{o n}}\left(\frac{k_{f_{l}}}{k_{p_{l}}}-n_{o f f} K_{I} g_{l} \frac{d}{d t}\right)}^{Q_{i l}} \Delta f_{l} \\
-\sum_{i=1}^{m_{i}}\left(1-q_{i}\right) \Delta P_{d c_{i}}^{r e f}+\sum_{i=1}^{m_{i}} q_{i} \sum_{j=1, j \notin A C_{i}}^{n_{o n}} \Delta P_{d c_{j}}^{r e f}
\end{gathered}
$$

for the onshore AC systems $i=1, \ldots, N$ and $q_{i}$ defined as $q_{i}=1 / k_{p_{i}} \sum_{j=1}^{n_{o n}} k_{p_{j}}$. The term $H_{i}^{\prime}$ corresponds to the effective inertia constant of system $i, C_{i}$ to the effective frequency droop in the system and $Q_{i l}$ to the coupling between frequencies in systems $i$ and $l$. Due to the presence of $f-V$ droop control, frequency variations in system $i$ will result in frequency changes in the other interconnected $\mathrm{AC}$ systems as well. An AC side frequency event in system $i$ is represented by $\Delta P_{L_{i}} \neq 0$ while a DC side event of converter $j$ by $\Delta P_{d c_{j}}^{r e f} \neq 0$.

Let us initially consider an AC side frequency event. From the above derivation we see that the inertial support from the offshore WFs $\left(K_{I}>0\right)$ increases the effective inertia of system $i$ by $\sum_{i=1}^{m_{i}} q_{i} n_{o f f} K_{I} g_{i}$. In addition, the terms $n_{o f f} K_{I} g_{l} d \Delta f_{l} / d t$ reduce the frequency variations in the interconnected $\mathrm{AC}$ systems caused by the frequency variation in system $i$. Therefore, the proposed WFS improves the transient frequency deviation of the onshore AC systems.

In case of a DC side frequency event in converter $j$ connected to the onshore system $i$, an opposite trend in the frequency variation of this system with respect to the rest of onshore systems is expected, as seen in the last two terms on the right hand side of equation (17) and explained in [12]. As mentioned before, the elements $g_{j}$ are greater or equal than 0 , avoiding the participation in over-frequency events, which means that the inertia contribution from the offshore WFs would have an opposite effect in this system as compared to the rest of AC systems. Depending on the outaged converter operating mode (rectifier/inverter) the benefit in minimizing the frequency fall is obtained by either increasing the system inertia or reducing the coupling between onshore systems.

Note that the above analysis is not dependant on the DC grid configuration and shows the benefit of WFS in a general framework. The simulation results shown later in Section VII are in agreement with this formulation.

\section{STUdy System}

The study system in Fig. 5 was adopted from [15] to illustrate the inertial support from offshore WFs connected through a DC grid. The 4-terminal DC grid interconnects three asynchronous AC systems: two onshore systems (System A and System B) and an offshore wind farm. System A is an interconnected AC system with two geographical regions [29] connected to the DC grid by converter stations $\# 2$ and \#3. System B is modeled by a single equivalent generator, G5, connected to station \#1. All the generators are conventional synchronous machines represented using the sub-transient model and equipped with both governor control (steam turbine) and excitation system (IEEE-DC1A type) [30].

The DC grid is a general asymmetric bipole grid as in [13]. We consider a lumped parameter pi-section model for the DC cable network, consisting of positive, negative and metallic return. The metallic return is only grounded at station \#1 with a $0.5 \Omega$ resistance and will carry the current in case of unbalanced operation in the system. The DC link voltage is $\pm 350 \mathrm{kV}$ and the $\mathrm{AC}$ systems are connected at $230 \mathrm{kV}$. Each converter station employs two 2-level VSC converters. Under nominal conditions, converter stations \#1 and \#4 act as rectifiers injecting active power into the DC grid, while \#2 and 


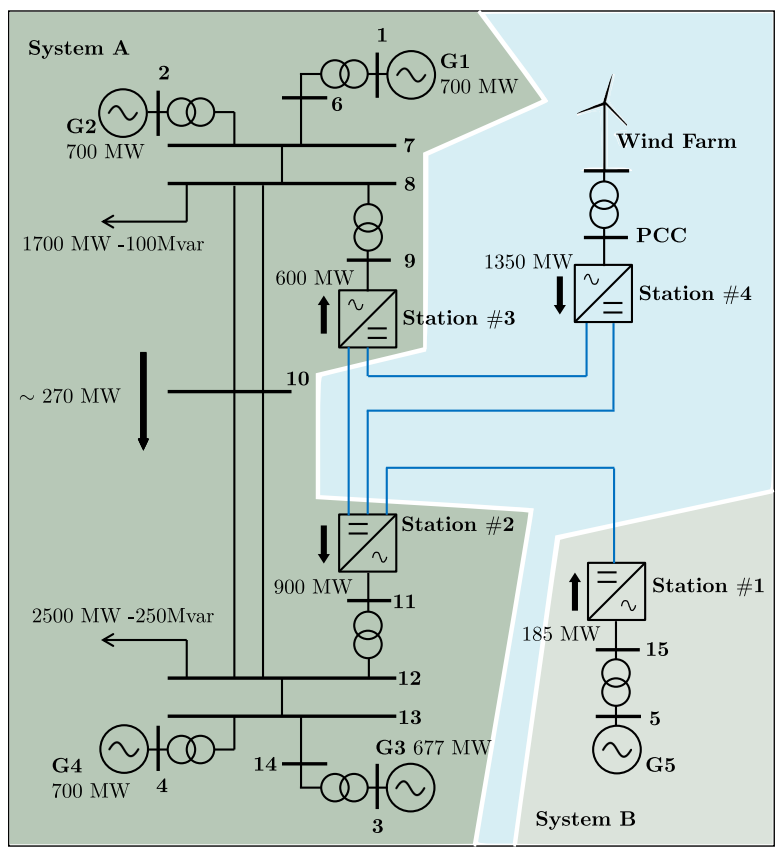

Fig. 5. 4-terminal DC grid interconnecting two onshore AC systems (Systems $\mathrm{A}$ and $\mathrm{B})$ and an offshore wind farm (WF).

\#3 act as inverters. An offshore wind farm (WF) with a rated capacity of $1350 \mathrm{MW}$ is connected at converter station \#4. An aggregated model of the WTGs within the WF was used in the simulation. The WTG parameters were obtained from a simulation model in DIgSILENT PowerFactory, corresponding to a 1.5 MW permanent magnet synchronous machine, with radius of $30 \mathrm{~m}, 18 \mathrm{rpm}$ rated speed and that enters the rated regime at $12.6 \mathrm{~m} / \mathrm{s}$. The following values were chosen in the simulations, conducted in DIgSILENT PowerFactory, for the droop controls in the onshore converters: $k_{p}=0.1, k_{f}=1.5$ and for the wind farm inertial controller: $K_{I}=50, T_{l p}=$ $1 s$. The droop constant values were chosen to strike a proper balance between conflicting objectives (adequate sensitivity vs. small DC link voltage variations) while ensuring the stability of the overall AC/DC grid system [13], [15].

\section{Simulation Results And Discussion}

The simulation results in this section are presented under three categories. First the performance of the CLS is compared against the proposed WFS in terms of power reference tracking. The effectiveness of the WFS to enable the inertial response from offshore WFs is then demonstrated for both AC side load change events and also sudden change in power reference of an onshore converter. Lastly, the performance of the WFS is validated under changing wind speed conditions.

\section{A. Tracking of Power Reference Changes}

For comparison, the CLS and the WFS were implemented separately at the offshore converter station \#4. A step in the power reference at both positive and negative pole converters was considered to increase the injected power from $185 \mathrm{MW}$ to $338 \mathrm{MW}$ in station \#1. The WF is assumed to operate in rated regime and produces $1350 \mathrm{MW}$.
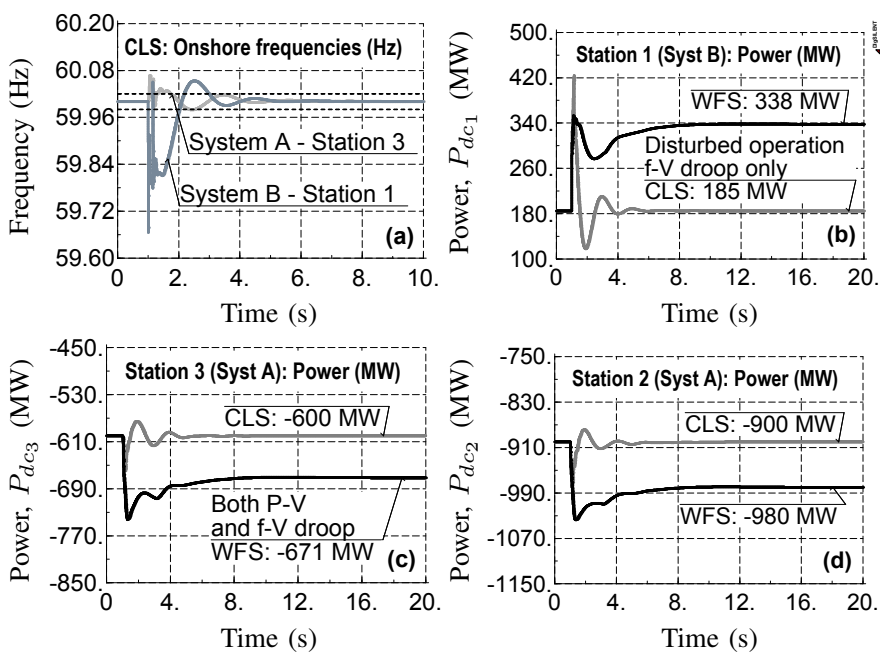

Fig. 6. System response after a step increase of real power reference at converter station \#1. (a) Onshore AC system frequency variations using the CLS. (b)-(d) Power injections into the DC grid. Gray trace: with CLS; Black trace: with WFS.

Figure 6(a) shows the frequency variation at the terminal of the onshore converter stations using the CLS. An opposing trend in the frequency variation of both AC systems can be observed which is typical for DC grid disturbances [12]. Clearly the CLS disturbed operation mode [17] (only $f$ - $V$ droop in place) is activated at all the onshore AC systems to maintain their corresponding frequency. It is to be noted that activation of the $f-V$ droop control (disturbed mode [17]) in one onshore system disturbs the frequency of the other onshore systems which could also activate their $f-V$ droop (and deactivate the $P-V$ droop control), as shown in Fig. 6(a). The real power variation at the converter stations using the CLS (gray traces) is compared against the WFS (black traces) in subplots (b)-(d) of Fig. 6. For the CLS strategy, as a result of the disturbed mode [17] activation, the power exchanges are held at the same values as they were before the step change in power reference command was provided, see Fig. 6(b)-(d) (gray traces). On the other hand, the proposed WFS alters the power exchanges in response to the step change command with each onshore converter sharing the burden equally (black traces). Note that the proposed WFS for inertial response from the WF is considered together with the presence of both $P$ $V$ and $f-V$ droop control loops at the onshore converters. The change in power reference required to achieve the desired power injection was factored into the control loops. Clearly, the ability of WFS to track power reference changes is one of the performance index for comparison against CLS.

\section{B. Load Change in AC Systems}

To demonstrate the effectiveness of inertial response using the WFS, a $40 \%$ step increase in the load connected at bus 8 in System A was considered. For this event the system response was investigated separately under high $(19 \mathrm{~m} / \mathrm{s})$ and moderate $(10 \mathrm{~m} / \mathrm{s})$ wind speed conditions. From here onwards, all the simulation results include $P-V$ droop control at the onshore converters. 

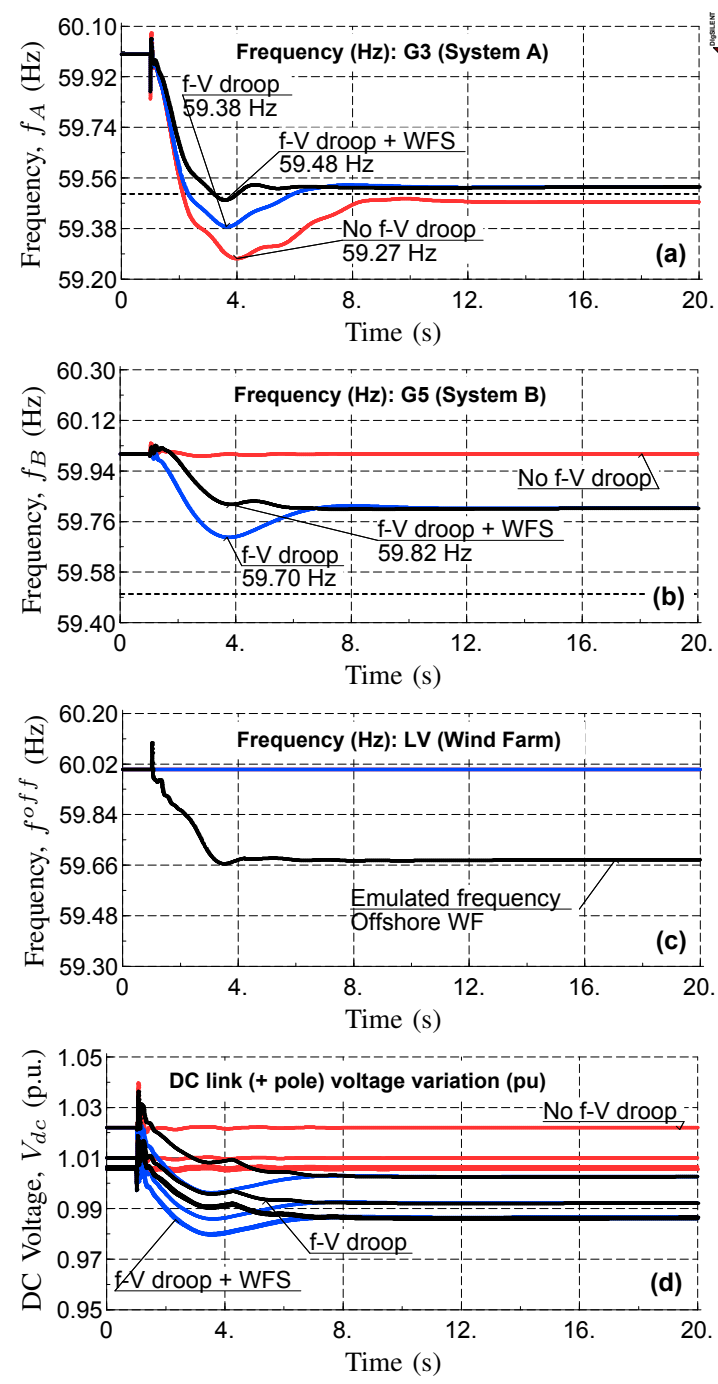

Fig. 7. System response after a step increase in load at bus 8 in System A under high wind speed condition. Red (light gray) trace: no $f-V$ droop control at onshore converter stations; Blue (dark gray) trace: with $f$ - $V$ droop control; Black trace: with $f-V$ droop and WFS for inertial response from WF.

1) High Wind Speed Condition: A constant $19 \mathrm{~m} / \mathrm{s}$ wind speed is considered under which the WF operates in the rated regime producing $1350 \mathrm{MW}$. The turbines operate with pitch control to provide the rated power and avoid over speeding.

The frequency variations in System A and B are shown, respectively, in subplots (a) and (b) of Fig. 7.

Without $f-V$ droop (for exchange of frequency support between AC systems) on onshore converters, the frequency of System A measured near slack generator $G 3$ (red traces) goes below $59.5 \mathrm{~Hz}$ with a maximum frequency excursion (frequency nadir) of $59.27 \mathrm{~Hz}$ (subplot (a)). The frequencies of System B and the offshore WF are virtually unaffected as those systems are decoupled from System A in absence of $f-V$ droop. With $f-V$ droop on the onshore converters the frequency variation in System A (blue trace) is less than before. However, this comes at the expense of an acceptable (less than $0.3 \mathrm{~Hz}$ ) frequency variation in System B. This is expected as the purpose of using the $f-V$ droop is to exchange frequency support among the onshore AC systems [14], [15].
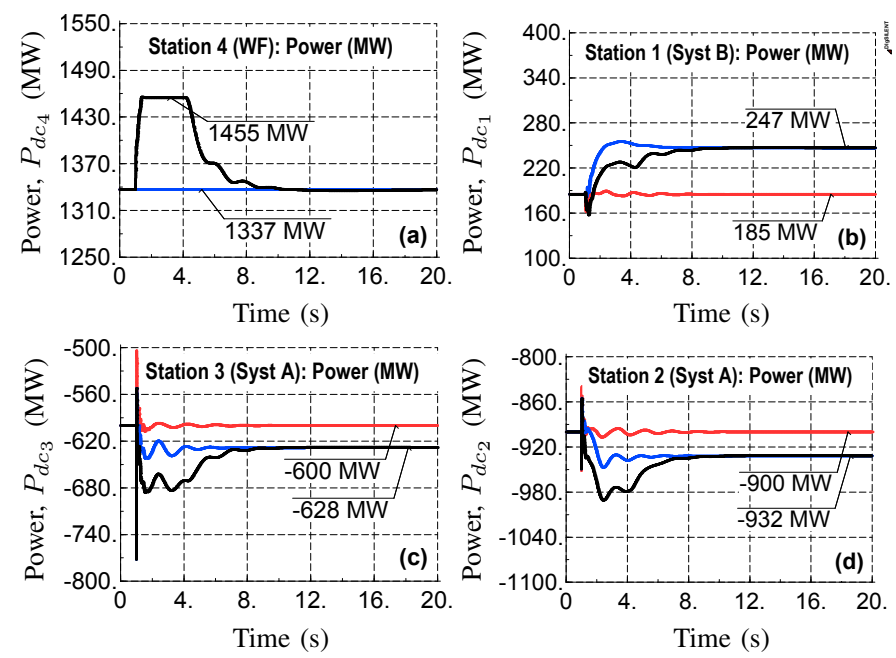

Fig. 8. Power injections into the DC grid after a step increase in load at bus 8 in System A under high wind speed condition. Red (light gray) trace: no $f$ - $V$ droop control at onshore converter stations; Blue (dark gray) trace: with $f-V$ droop control; Black trace: with $f-V$ droop and WFS for inertial response from WF.

Activation of the WTG control for inertial response using the WFS improves the transient variation of the frequency (black trace) for both Systems A and B. Both the frequency nadir and the rate of change of frequency $(\mathrm{RoCoF})$ are reduced. This is enabled by a properly emulated offshore frequency variation as shown by the black trace in Fig. 7(c) which closely resembles the actual frequency variations (black traces) in Systems A (Fig. 7(a)) and B (Fig. 7(b)). The associated DC link voltage variations, shown in Fig. 7(d) are within the acceptable limits.

The frequency variations in Fig. 7 are driven by the power exchanges with the DC grid shown in Fig. 8. In the absence of $f-V$ droop control (red traces), the power exchanged between the DC grid and the AC systems is not affected as they are decoupled. With only $f-V$ droop control activated (blue traces) more power is injected through stations \#2 and \#3 into System A to supply the increased load. With a constant WF output (control for inertial response is not active), this extra power comes from System B.

When both $f-V$ droop control on onshore converters and the inertial control in the WTGs (black traces) are active, the release of inertial power (as shown in Fig. 8(a)) relieves the burden on System B by increasing the power injection into System A during the transient period, which improves the frequency variations in Fig. 7(a), (b).

The aggregated response of the WF is plotted in Fig. 9. The inertial power is extracted by reducing the pitch angle (subplot (b)) with only a small change in the rotational speed of the turbine (subplot (c)).

Following inertial response the power output of the WF returns to the pre-disturbance level (subplot (a)). The amount of such temporary over production would be limited by the short-term overload capability of the WTG components, especially the converters, and also by the DC grid converters. For this exercise, a $9 \%$ overload capacity in the tens of seconds time frame was assumed for the converters in the WT and the 

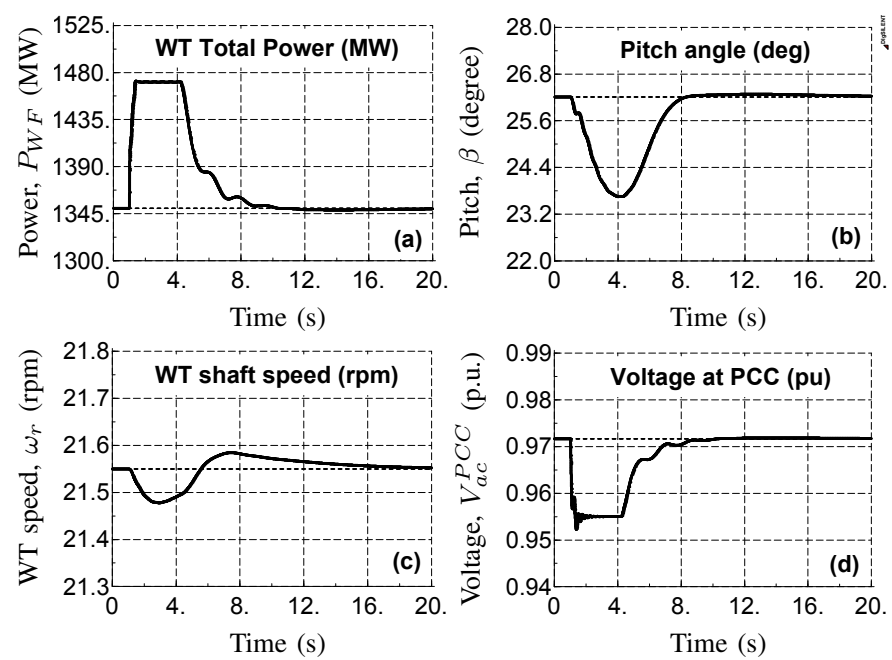

Fig. 9. Wind farm (WF) response with $f$ - $V$ droop and WFS for inertial response under high wind speed condition.

DC grid.

2) Moderate Wind Speed Condition: The same load event of the previous section is considered here under a moderate wind speed of $10 \mathrm{~m} / \mathrm{s}$. The WF operates in sub-rated regime and produces $680 \mathrm{MW}$. The turbine is controlled to operate at maximum power coefficient $C_{p}$ while the pitch angle is kept constant at $0^{\circ}$.

As in the previous case the effectiveness of inertial control using the WFS is evident both in terms of improved RoCoF and frequency nadir as shown in Fig. 10. However, due to the recovery phase of the wind turbines, the restoration of postevent steady state is slower with a slight decrease of frequency observed after the initial support.

The response of the WF operating in sub-rated regime is shown in Fig. 11. The inertial control extracts additional power from the WF by slowing down the turbines (subplot (c)). With decrease of the turbine speed, the MPPT control limits the turbine deceleration by reducing the power reference (subplot (b). Following the inertial support, there is a recovery phase before the turbines regain their original operating point after about 40s.

Note that we have considered an aggregated model of the WTGs to model the WF. In reality, the wind speed across all the turbines in the WF is not uniform due to wake effect [31]. As the considered inertial control is implemented at the wind turbine level, the individual WTG will react to frequency changes in its collection network to provide inertial support, experiencing different recovery periods depending on the wind speed present at the turbine.

\section{Change of Converter Power Reference}

In the previous section the effectiveness of inertial control using the WFS was illustrated for a load event within an onshore AC system. Here a disturbance within the DC grid is considered in the form of a step increase in the power reference at converter station \#1, same as in Section VII-A. The WF is assumed to operate at the rated regime producing $1350 \mathrm{MW}$.
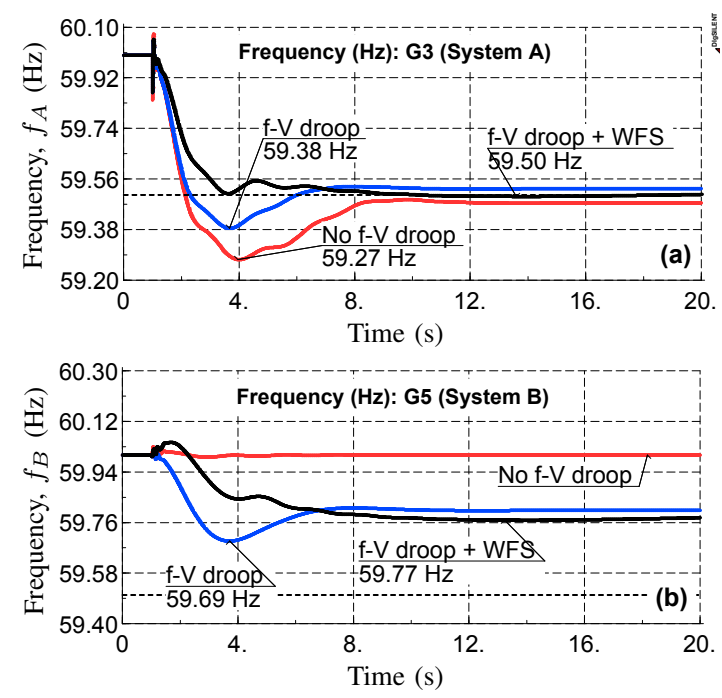

Fig. 10. Onshore AC system frequency variations after a step increase in load at bus 8 in System A under moderate wind speed condition. Red (light gray) trace: no $f$ - $V$ droop control at onshore converter stations; Blue (dark gray) trace: with $f-V$ droop control; Black trace: with $f-V$ droop and WFS for inertial response from WF.
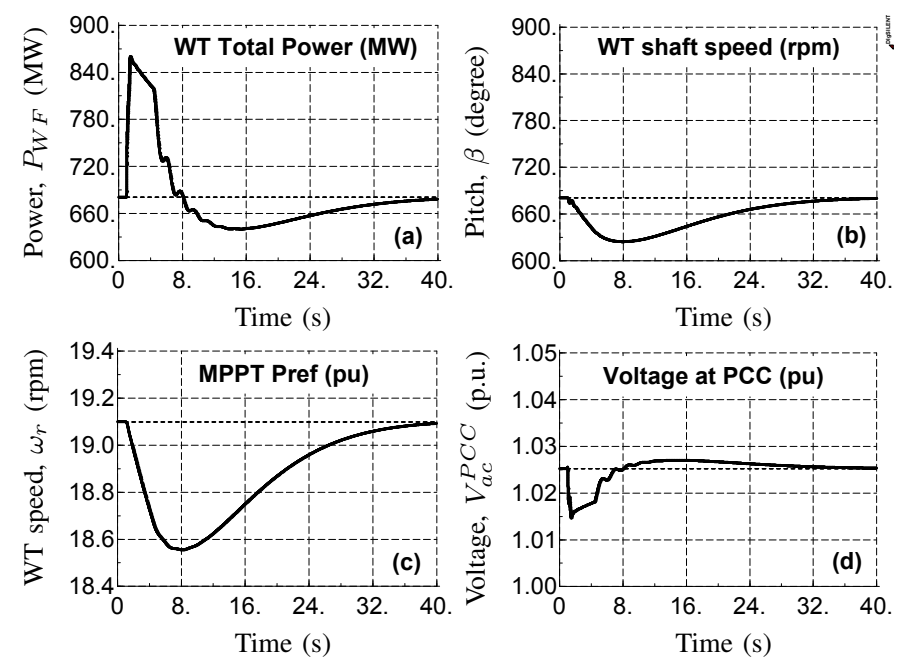

Fig. 11. Wind farm (WF) response with $f-V$ droop and WFS for inertial response under moderate wind speed condition.

The system responses are shown in Fig. 12. As a result of the increased power export out of System B its frequency goes down as shown in subplot (b). The frequency of System A (near generator G3) increases as shown in subplot (a). This opposite trend in frequency variations is typical for DC grid disturbances as already mentioned in Section VII-A.

The inertial support provided by the WF (black traces) improves the frequency nadir in System B compared to the case where only $f-V$ droop control is active on onshore converters (blue traces), see subplot (b). However, the additional inertial power injected by the WF causes a slight increase in the frequency of System A which is expected due to exchange of frequency support enabled by the $f-V$ droop control on onshore converters. In this case, the frequency variation in System B is emulated at the offshore WF (see subplot (c)) with no contribution from the positive frequency variation 

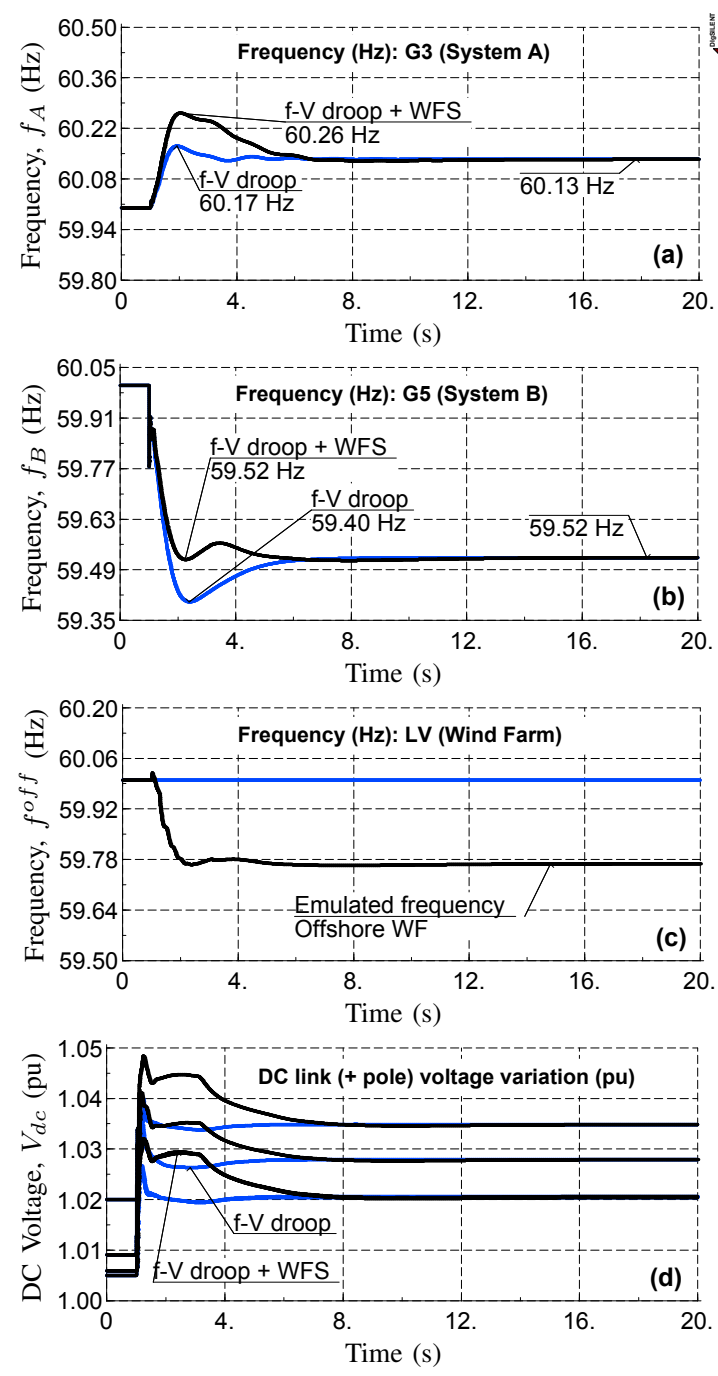

Fig. 12. System response after a step increase of real power reference at converter station \#1 under high wind speed condition. Blue (dark gray) trace: with $f-V$ droop control at onshore converter stations; Black trace: with $f-V$ droop and WFS for inertial response from WF.

$\left(f_{j}-f_{j}^{r e f}\right)$ in System A.

\section{Change of Wind Speed}

A sudden change in the wind speed results in variations of onshore AC system frequencies which would subsequently trigger inertial response from the WFs. To validate the control performance and the system transient response, we consider step changes to vary the wind speed. Although wind speed variations might be slower, step response simulations are interesting as they significantly affect the frequency of the onshore systems. The system response to such step changes in wind speed is shown in Fig. 13.

The presence of the inertial control using the WFS (black traces) improves the RoCoF and frequency nadir (which is not clear due to relatively long time scale) for both $\mathrm{AC}$ systems (System A and B) after the wind speed changes, see Fig. 13(a), (b). This is achieved by reducing the turbine speed to provide the inertial support (subplot (c)). It is to be noted that the WTG control adapts the rotor speed according to the wind speed to
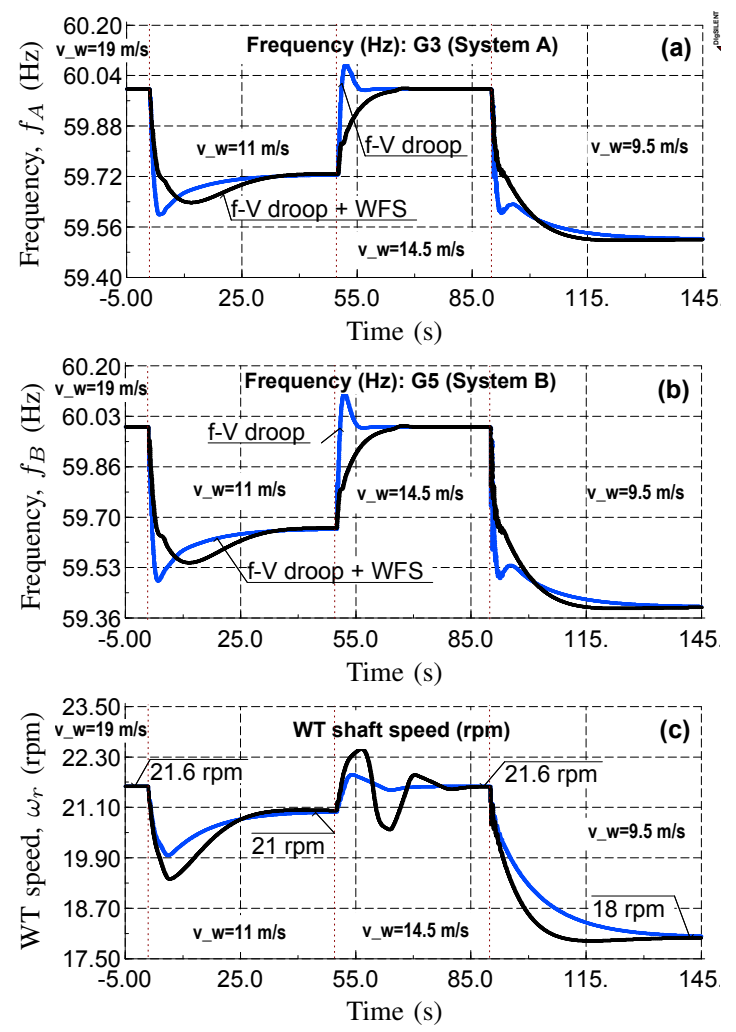

Fig. 13. System response to wind speed changes. Blue (dark gray) trace: with $f$ - $V$ droop control at onshore converter stations but no inertial response from WF; Black trace: with $f-V$ droop and WFS for inertial response from WF.

extract either the maximum power (sub-rated regime) or to operate at its rated value (rated regime), see subplot (c). For sub-rated regime $\left(v_{w}<12.6 \mathrm{~m} / \mathrm{s}\right)$, there is a recovery period following the inertial response which causes the frequency to go slightly below the one obtained with only $f-V$ droop control.

\section{CONCLUSION}

A methodology for providing inertial response from offshore wind farms connected through a DC grid is demonstrated. As an extension to a communication-less scheme reported in the literature, this paper adopts an approach where the variations in onshore system frequencies are communicated to the offshore converters using the fibre optic link embedded within the sub-sea DC cables. The analytical formulation presented in this paper shows the effective change in system inertia and frequency droop as a result of inertial support using the proposed WFS strategy. The case study on a 4 terminal DC grid connecting an offshore wind farm and two onshore AC systems also illustrates the inertial support from offshore WFs with the considered methodology. Different frequency events within the AC system and the DC grid were studied for varying wind speed conditions.

It is shown that the proposed WFS approach can improve the transient frequency deviation in the AC systems experiencing under-frequency problems which is critical for secure operation of low inertia systems of the future. 


\section{REFERENCES}

[1] (2012) Electricity Ten Year Statement (TYS). National Grid. [Online]. Available: http://www.nationalgrid.com/uk/Electricity/tenyear-statement/current-elec-tys/

[2] G. Stein. (2011) Frequency Response Technical Sub-Group Report. National Grid. [Online]. Available: http://www.nationalgrid.com/NR/rdonlyres/2AFD4C05-E1694636-BF02-EDC67F80F9C2/50090/FRTSGGroupReportFinal.pdf

[3] J. Ekanayake and N. Jenkins, "Comparison of the response of doubly fed and fixed-speed induction generator wind turbines to changes in network frequency," Energy Conversion, IEEE Transactions on, vol. 19, no. 4, pp. 800-802, 2004.

[4] G. Lalor, A. Mullane, and M. O'Malley, "Frequency control and wind turbine technologies," Power Systems, IEEE Transactions on, vol. 20, no. 4, pp. 1905-1913, Nov 2005.

[5] Z. Miao, L. Fan, D. Osborn, and S. Yuvarajan, "Wind farms with HVdc delivery in inertial response and primary frequency control," Energy Conversion, IEEE Transactions on, vol. 25, no. 4, pp. 1171-1178, Dec 2010.

[6] Y. Phulpin, "Communication-free inertia and frequency control for wind generators connected by an HVDC-link," Power Systems, IEEE Transactions on, vol. 27, no. 2, pp. 1136-1137, May 2012.

[7] Y. Pipelzadeh, B. Chaudhuri, and T. C. Green, "Inertial response from remote offshore wind farms connected through VSC-HVDC links: A communication-less scheme," in Power and Energy Society General Meeting, 2012 IEEE, July 2012, pp. 1-6.

[8] T. M. Haileselassie, R. E. Torres-Olguin, T. K. Vrana, K. Uhlen, and T. Undeland, "Main grid frequency support strategy for VSC-HVDC connected wind farms with variable speed wind turbines," in PowerTech, 2011 IEEE Trondheim, June 2011, pp. 1-6.

[9] N. Flourentzou, V. G. Agelidis, and G. D. Demetriades, "VSC-Based HVDC power transmission systems: An overview," Power Electronics, IEEE Transactions on, vol. 24, no. 3, pp. 592-602, 2009.

[10] J. Blau, "Europe plans a North Sea grid," Spectrum, IEEE, vol. 47, no. 3, pp. 12-13, 2010.

[11] S. Gordon, "Supergrid to the rescue," Power Engineer, vol. 20, no. 5, pp. 30-33, 2006.

[12] I. Martínez Sanz, B. Chaudhuri, and G. Strbac, "Frequency changes in AC systems connected to DC grids: Impact of AC vs. DC side events," in Power and Energy Society General Meeting, 2014 IEEE, July 2014, pp. $1-5$.

[13] N. R. Chaudhuri and B. Chaudhuri, "Adaptive droop control for effective power sharing in multi-terminal DC (MTDC) grids," Power Systems, IEEE Transactions on, vol. 28, no. 1, pp. 21-29, Feb 2013.

[14] T. M. Haileselassie and K. Uhlen, "Primary frequency control of remote grids connected by multi-terminal HVDC," in Power and Energy Society General Meeting, 2010 IEEE, July 2010, pp. 1-6.

[15] N. R. Chaudhuri, R. Majumder, and B. Chaudhuri, "System frequency support through multi-terminal DC (MTDC) grids," Power Systems, IEEE Transactions on, vol. 28, no. 1, pp. 347-356, 2013.

[16] J. Dai, Y. Phulpin, A. Sarlette, and D. Ernst, "Coordinated primary frequency control among non-synchronous systems connected by a multiterminal high-voltage direct current grid," Generation, Transmission Distribution, IET, vol. 6, no. 2, pp. 99-108, February 2012.

[17] B. Silva, C. L. Moreira, L. Seca, Y. Phulpin, and J. A. Peças Lopes, "Provision of inertial and primary frequency control services using offshore multiterminal HVDC networks," Sustainable Energy, IEEE Transactions on, vol. 3, no. 4, pp. 800-808, 2012.

[18] J. Beerten and R. Belmans, "Analysis of power sharing and voltage deviations in droop-controlled DC grids," Power Systems, IEEE Transactions on, vol. 28, no. 4, pp. 4588-4597, Nov 2013.

[19] S. Zhou, J. Liang, J. B. Ekanayake, and N. Jenkins, "Control of multiterminal VSC-HVDC transmission system for offshore wind power generation," in Universities Power Engineering Conference (UPEC), 2009 Proceedings of the 44th International, Sept 2009, pp. 1-5.

[20] X. Hu, J. Liang, D. J. Rogers, and Y. Li, "Power flow and power reduction control using variable frequency of offshore AC grids," Power Systems, IEEE Transactions on, vol. 28, no. 4, pp. 3897-3905, Nov 2013.

[21] A. Madariaga, J. Martín, I. Zamora, I. M. de Alegría, and S. Ceballos, "Technological trends in electric topologies for offshore wind power plants," Renewable and Sustainable Energy Reviews, vol. 24, pp. 3244, 2013.

[22] R. G. de Almeida and J. A. Peças Lopes, "Participation of doubly fed induction wind generators in system frequency regulation," Power Systems, IEEE Transactions on, vol. 22, no. 3, pp. 944-950, 2007.
[23] N. R. Ullah, T. Thiringer, and D. Karlsson, "Temporary primary frequency control support by variable speed wind turbines; potential and applications," Power Systems, IEEE Transactions on, vol. 23, no. 2, pp. 601-612, May 2008.

[24] J. Conroy and R. Watson, "Frequency response capability of full converter wind turbine generators in comparison to conventional generation," Power Systems, IEEE Transactions on, vol. 23, no. 2, pp. 649-656, May 2008.

[25] J. Morren, J. Pierik, and S. W. de Haan, "Inertial response of variable speed wind turbines," Electric Power Systems Research, vol. 76, no. 11, pp. 980-987, 2006 .

[26] J. M. Mauricio, A. Marano, A. Gómez-Expósito, and J. L. Martínez Ramos, "Frequency regulation contribution through variablespeed wind energy conversion systems," Power Systems, IEEE Transactions on, vol. 24, no. 1, pp. 173-180, Feb 2009.

[27] I. D. Margaris, S. A. Papathanassiou, N. D. Hatziargyriou, A.-D. Hansen, and P. Sørensen, "Frequency control in autonomous power systems with high wind power penetration," Sustainable Energy, IEEE Transactions on, vol. 3, no. 2, pp. 189-199, April 2012.

[28] S. Dodds, B. Railing, K. Akman, B. Jacobson, T. Worzyk, and B. Nilsson, "HVDC VSC (HVDC light) transmission operating experiences," in Cigre 2010 session, August 2010, pp. 1-9.

[29] P. Kundur, Power System Stability and Control. McGraw-Hill, 1993.

[30] "IEEE recommended practice for excitation system models for power system stability studies," IEEE Std 421.5-2005 (Revision of IEEE Std 421.5-1992), pp. 1-85, 2006.

[31] S. Kuenzel, L. P. Kunjumuhammed, B. C. Pal, and I. Erlich, "Impact of wakes on wind farm inertial response," Sustainable Energy, IEEE Transactions on, vol. 5, no. 1, pp. 237-245, Jan 2014.

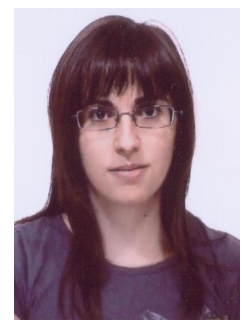

Inmaculada Martínez Sanz (S'13) received the electrical engineering degree from University of Seville, Seville, Spain in 2010. She is currently a Ph.D. student at Imperial College London, London, U.K. Her research interests include power systems dynamics and control, HVDC transmission and wind power.

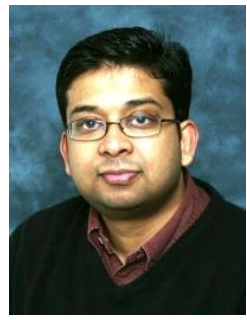

Balarko Chaudhuri (M'06 - SM'11) received the $\mathrm{Ph} . \mathrm{D}$. degree in 2005 from Imperial College London, London, U.K. He is presently a Senior Lecturer in the Control and Power Research Group, Imperial College London. His area of interest is electric power transmission systems, control theory, smart grids and renewable energy. Dr. Chaudhuri is a member of the IET and Cigré. He is an Associate Editor of IEEE SYSTEMS JOURNAL and Elsevier Control Engineering Practice.

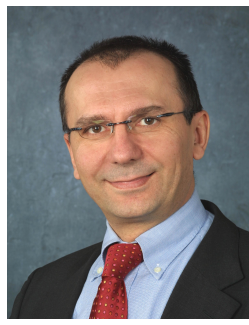

Goran Strbac (M'95) is a Professor of Electrical Energy Systems with Imperial College London, London, U.K. His research interests are in modeling and optimization of electricity system operation and investment, economic and pricing, including integration of new forms of generation and demand side technologies. 